ORIGINAL ARTICLE

\title{
Communication failures in the operating room: an observational classification of recurrent types and effects
}

\author{
L Lingard, S Espin, S Whyte, G Regehr, G R Baker, R Reznick, J Bohnen, B Orser, D Doran, \\ E Grober
}

See end of article for authors' affiliations

Correspondence to: L Lingard, PhD, Associate Professor, Department of Paediatrics, University of Toronto Faculty of Medicine, Donald R. Wilson Centre for Research in Education, University Health Network, 200 Elizabeth Street, Eaton South 1-604, Toronto, Ontario, Canada M5G 2C4; lorelei. lingard@utoronto.ca

Accepted for publication 11 March 2004
Background: Ineffective team communication is frequently at the root of medical error. The objective of this study was to describe the characteristics of communication failures in the operating room (OR) and to classify their effects. This study was part of a larger project to develop a team checklist to improve communication in the OR.

Methods: Trained observers recorded 90 hours of observation during 48 surgical procedures. Ninety four team members participated from anesthesia (16 staff, 6 fellows, 3 residents), surgery (14 staff, 8 fellows, 13 residents, 3 clerks), and nursing (31 staff). Field notes recording procedurally relevant communication events were analysed using a framework which considered the content, audience, purpose, and occasion of a communication exchange. A communication failure was defined as an event that was flawed in one or more of these dimensions.

Results: 421 communication events were noted, of which 129 were categorized as communication failures. Failure types included "occasion" (45.7\% of instances) where timing was poor; "content" $(35.7 \%)$ where information was missing or inaccurate, "purpose" $(24.0 \%)$ where issues were not resolved, and "audience" (20.9\%) where key individuals were excluded. $36.4 \%$ of failures resulted in visible effects on system processes including inefficiency, team tension, resource waste, workaround, delay, patient inconvenience and procedural error.

Conclusion: Communication failures in the OR exhibited a common set of problems. They occurred in approximately $30 \%$ of team exchanges and a third of these resulted in effects which jeopardized patient safety by increasing cognitive load, interrupting routine, and increasing tension in the OR.
$\mathrm{R}$ ecent evidence suggests that adverse events resulting from error happen at unacceptably high rates in the inpatient setting ${ }^{1}$ and that ineffective or insufficient communication among team members is often a contributing factor. ${ }^{23}$ In fact, communication failures have been uncovered at the root of over $60 \%$ of sentinel events reported to the Joint Commission on Accreditation of Healthcare Organizations. ${ }^{4}$ Coroner reports also expose the role of communication in error: a recent inquest report cited "communications difficulties at all levels of the hospital, including doctors to doctors, doctors to nurses, nurses to nurses and nurses to doctors" as the primary cause of errors leading to the death of a paediatric patient. ${ }^{5}$

There is a growing literature on the critical relationship between teamwork and safety in health care. ${ }^{67}$ The trend in this literature is towards studying teamwork as a cluster of behaviours-for example, leadership, technical skills, coordination, situational awareness, communication-and producing multidimensional schemes to capture the quality of teamwork. $^{8-10}$ While these models have reinforced the importance of communication in effective team function, their multidimensionality precludes in depth attention to the individual variable of communication. Theory based attention to communication is necessary to sharpen current insights regarding its critical role in teamwork and safety.

Operating room (OR) teams have been the focus of much recent research, including sociological and human factors descriptions of teamwork; ${ }^{2}$ survey research reporting the attitudes of OR personnel towards teamwork, communications, and leadership; ${ }^{3}$ management studies of team routine; ${ }^{11}$ analyses of the relationship between behavioral markers and safe outcomes; ${ }^{8}$ and ethnographic explorations of communication patterns and interpretive perceptions. ${ }^{12}{ }^{13}$

This last ethnographic work offered the first descriptive categorization of common OR team communication behaviors as observed in their natural setting and interpreted by participants (surgeons, nurses, anesthesiologists) in focus group sessions. Communication patterns were observed to be variable from case to case and team to team. Critical information was often transferred in an ad hoc reactive manner and tension levels were frequently high. Interviewed team members varied in their perceptions of team roles and motivations underlying communication events, while they agreed that communicative tension negatively affects administrative, educational, and clinical outcomes.

These findings suggest that the current weaknesses in communication in the OR may derive from a lack of standardization and team integration. Team members do not commonly convene to discuss key issues before a case, decisions are often made without all relevant team members present, and much communication is consequently reactive and tension provoking. Statistical insurance claims data support these findings: in a review of closed surgery claims from 1991 to 2000 the Controlled Risk Insurance Company (CRICO) identified inadequate information sharing among team members as a primary trigger for claims and reported that $15 \%$ of claim cases included a "communication breakdown". ${ }^{14}$

One potential solution to the described weaknesses in OR team communication is to adapt the checklist system currently in use for systematic preflight team communications in the aviation industry. ${ }^{15}$ The aviation checklist 
structures the communication of critical information to ensure that all team members possess accurate and explicit data and decisions are made in a context where cross checking can occur. While the OR and the cockpit are clearly distinct domains, we anticipate that a carefully adapted checklist system could promote safer, more effective communications in the OR team. Discipline specific checklists already serve important safety functions in the OR-for example, the pre-anesthetic equipment checklist is considered a standard of practice by the Canadian and American Societies of Anesthesiology. ${ }^{16}$ The key feature of the preoperative team checklist system is that it would ensure the exchange of pertinent information among all OR team members; it would supplement rather than supplant existing communication practices within each discipline-for example, surgeon-surgical resident or nurse-nurse discussions.

Effective adaptation of the checklist system for the OR environment requires in depth understanding of the critically relevant information that would optimally be communicated to the team before a surgical procedure. It also requires classification of the current patterns of weakness or failure in this communication process, as well as the outcome dimensions that could be measured following a checklist intervention. This study sought to describe systematically the content and effects of procedurally relevant communication eventsthat is, any communication relevant to the surgical procedure itself and excluding social conversations and other discourse not immediately germane to the team's procedural tasks. The study also sought to define and classify common communication failures.

\section{METHODS}

\section{Research setting}

Following approval from the hospital research ethics board, consent for study participation was obtained from a convenience sample of OR team members in the divisions of general and vascular surgery. Previous research has shown that these divisions are representative of a range of communication patterns and approaches. ${ }^{12} 13$

\section{Data collection}

Over 3 months in the winter of 2003, 90 hours of observation were conducted during 48 procedures in general and vascular surgery. Procedures were purposively sampled to represent a range of surgical cases and included breast, thyroid, colorectal, hepatobiliary, vascular, transplant, and laparoscopic surgery. Ninety four team members were observed, including 16 anesthesia staff, six anesthesia fellows, three anesthesia residents, 14 surgical staff, eight surgical fellows, 13 surgical residents, three clinical clerks, and 31 nurses. No team members declined to participate. While some complete cases were observed, most observations focused on the first 2 hours of a case (including preparation, administration of anesthesia, and opening), during which the majority of procedurally relevant team communication occurs. Ethnographic field note methods ${ }^{17}$ were used to record communication events including time of event, participants, content, contextual features (such as what team members were engaged in during the event) and, if available to the observer, any immediate visible effects. A communication event was defined as a verbal or non-verbal exchange between two or more team members. The three observers were well trained in field methods and had a critical combination of relevant skills including OR nursing background, communications expertise, and experience with observational research.

\section{Data analysis}

Field notes were analysed in a constant comparative manner by three researchers to identify failures in communication events among team members. ${ }^{18}$ The constant comparative approach involves iterative reading of field notes, comparing any instance of interest with others, both similar and different, in order to develop a sense of pattern and relations. Analysts alternated independent analysis with group discussion. Two phases of analysis were employed. Firstly, a rhetorical framework was used to define the parameters of communication failure. This framework, which is particularly useful for examining group discourse in complex social settings, considers content of communication alongside three other critical factors: audience, purpose, and occasion. ${ }^{19} 20$ "Audience" refers to the participants present during an exchange; "purpose" refers to the goals, implicit or explicit, of the communication; and "occasion" refers to the physical and temporal situation of an exchange. A communication failure was defined as an event that was flawed in terms of one or more of these rhetorical factors. For instance, if a request was made of the wrong team member, this would be categorized as an "audience" flaw. Similarly, if a comment was rendered inaudible by an alarm, this would be categorized as an "occasion" flaw. In the second phase of the analysis instances organized within each of these four rhetorical categories were analysed for trends in type of exchange and effects on system processes. To ensure trustworthiness of analysis, local experts familiar with the OR work environment reviewed samples of analysed data. Discrepancies were resolved by discussion of the context and content of a failure, comparison with other instances in a potential category, and return to the field notes for further information.

\section{RESULTS}

\section{Types of communication failure}

Analysis of the field notes produced by observers yielded records of 421 procedurally relevant communication events. Some events were brief-such as a question and response sequence involving two team members-while others were more enduring and inclusive-such as a discussion among members of all three team disciplines about patient blood loss during a critical period of the procedure. Of these $42 \mathrm{l}$ events, 129 were categorized as communication failures related to one or more of the rhetorical factors described above (table 1). The four rhetorical factors provided an effective framework for detecting and categorizing communication failures; no observed exchange fell outside this framework.

Within each rhetorical factor, observed failures exhibited one or two recurrent types of exchange. Table 2 defines each category of communication failure and provides an excerpt from the field notes illustrating the category's dominant exchange type. Of the four types, the most common communication failure was "occasion" (45.7\% of instances). All events in this category involved suboptimal timing of an

Table 1 Summary of communication events recorded and classification of communication failures

\begin{tabular}{ll}
\hline Communication events recorded ( $n$ ) & 421 \\
$\begin{array}{l}\text { Communication events classified as } \\
\text { communication failures (\% of total events) }\end{array}$ & $129(30.6)$ \\
$\begin{array}{l}\text { Communication failures by type (\% of total } \\
\text { of communication failures) }\end{array}$ \\
Occasion & $59(45.7)$ \\
Content & $46(35.7)$ \\
Purpose & $31(24.0)$ \\
Audience & $27(20.9)$ \\
\hline & \\
*Because a single communication event could be classified \\
within more than one category of rhetorical failure, numbers \\
add up to more than 100\%.
\end{tabular}


Table 2 Definitions of types of communication failure with illustrative examples and notes

\begin{tabular}{|c|c|c|}
\hline Failure & Definition & Illustrative example and analytical note (in italics) \\
\hline Occasion failures & $\begin{array}{l}\text { Problems in the situation or context of the } \\
\text { communication event }\end{array}$ & $\begin{array}{l}\text { The staff surgeon asks the anesthesiologist whether the antibiotics have been } \\
\text { administered. At the point of this question, the procedure has been underway for over } \\
\text { an hour. } \\
\text { Since antibiotics are optimally given within } 30 \text { minutes of incision, }{ }^{21} \text { the timing of this } \\
\text { inquiry is ineffective both as a prompt and as a safety redundancy measure. }\end{array}$ \\
\hline Content failures & $\begin{array}{l}\text { Insufficiency or inaccuracy apparent in the } \\
\text { information being transferred }\end{array}$ & $\begin{array}{l}\text { As the case is set up, the anesthesia fellow asks the staff surgeon if the patient has an ICU } \\
\text { (intensive care unit) bed. The staff surgeon replies that the "bed is probably not needed, } \\
\text { and there isn't likely one available anyway, so we'll just go ahead." } \\
\text { Relevant information is missing and questions are left unresolved: has an ICU bed been } \\
\text { requested, and what will the plan be if the patient does need critical care and an ICU } \\
\text { bed is not available? [Note: this example was classified as both a content and a purpose } \\
\text { failure.] }\end{array}$ \\
\hline Audience failures & $\begin{array}{l}\text { Gaps in the composition of the group engaged } \\
\text { in the communication }\end{array}$ & $\begin{array}{l}\text { The nurses and anesthesiologist discuss how the patient should be positioned for surgery } \\
\text { without the participation of a surgical representative. } \\
\text { Surgeons have particular positioning needs so they should be participants in this } \\
\text { discussion. Decisions made in their absence occasionally lead to renewed discussions } \\
\text { and repositioning upon their arrival. }\end{array}$ \\
\hline Purpose failures & $\begin{array}{l}\text { Communication events in which purpose is } \\
\text { unclear, not achieved, or inappropriate }\end{array}$ & $\begin{array}{l}\text { During a living donor liver resection, the nurses discuss whether ice is needed in the } \\
\text { basin they are preparing for the liver. Neither knows. No further discussion ensues. } \\
\text { The purpose of this communication-to find out if ice is required-is not achieved. No } \\
\text { plan to achieve it is articulated. }\end{array}$ \\
\hline
\end{tabular}

exchange such that information was requested or provided too late to be maximally useful. The "content" category (35.7\% of instances) included two types of exchange-those in which relevant information was missing which accounted for the majority of events in this category, and those in which inaccurate information was exchanged. The "purpose" category $(24.0 \%$ of instances) also included two types of exchange. These communication failures related predominantly to participants' failure to achieve communicative objectives due to lack of resolution of an issue raised, but also included a small number of events where the objective was interpreted by observers as inappropriate (for example, provocation of another team member). Finally, "audience" failures (20.9\% of instances) all involved the absence of a key team member during the communication event, most frequently the absence of a surgical representative in discussions regarding the preparation for surgery such as the set up of equipment and the positioning and draping of the patient.

\section{Effects of communication failures}

Each instance of communication failure was further examined to determine whether it resulted in a visible effect and to describe the nature of those effects. 36.4\% of communication failures resulted in visible effects on system processes which included inefficiency, team tension, resource waste, workaround, delay, patient inconvenience, and procedural error. The remainder of failures resulted in no visible immediate effect. Table 3 summarizes the distribution of effects and table 4 defines each effect type and provides an illustrative excerpt from the field notes.

The types of communication failure most likely to result in an observable effect were "occasion" (55.9\% of failures linked to effect) and "purpose" (45.5\% of failures linked to effect). Relatively fewer effects were linked to "audience" and "content" failures $(25.0 \%$ and $22.2 \%$, respectively). "Occasion" failures led most frequently to inefficiency, team tension, and delay, while "purpose" failures were associated with only two effect types: inefficiency and tension.

\section{DISCUSSION}

Communication failures on the operating team are frequent, occurring in approximately $30 \%$ of procedurally relevant

\begin{tabular}{|c|c|}
\hline Effect of communication failure by type & No $(\%)^{*}$ \\
\hline $\begin{array}{l}\text { No visible immediate effect } \\
\text { Inefficiency } \\
\text { Team tension } \\
\text { Delay } \\
\text { Workaround } \\
\text { Resource waste } \\
\text { Patient inconvenience } \\
\text { Procedural error }\end{array}$ & $\begin{array}{l}82(63.6) \\
23(17.8) \\
16(12.3) \\
10(7.7) \\
3(2.3) \\
2(1.6) \\
2(1.6) \\
1(0.8)\end{array}$ \\
\hline
\end{tabular}

exchanges among team members. More encouragingly, we have found that these failures are based in strikingly simple factors: communication is too late to be effective, content is not consistently complete and accurate, key individuals are excluded, and issues are left unresolved until the point of urgency. Parallels between these factors and the principles of the aviation checklist system-to communicate proactively, with complete and accurate data, to all relevant team members in order to achieve explicit and shared goalsunderscore the suitability of such an intervention for improving OR team communication.

The results of this study may be affected by the potential for sampling bias among the OR team participants. That no team members declined participation suggests a low likelihood of such bias; however, to the extent that participants may have been unusually interested or confident in their communication abilities, the results presented in this paper may represent a "tip of the iceberg" in terms of the nature and frequency of communication failure and its effects. Assessing the transferability of these findings to other OR teams in other institutional settings requires further research.

Intervening to strengthen communicative practice among healthcare teams is complicated because such communication is rooted in the distinct and often conflicting professional identities of team members and is bounded by a culture that has been traditionally and persistently hierarchical. However, notwithstanding the complexity of interprofessional communication, our descriptive classification of 
Table 4 Definitions of effect types with illustrative examples and notes

Effect and definition
Inefficiency:
Communication failure requires team members to
redo or undo a procedural step; step requires more
actions or discourse than usual

\section{Illustrative example and analytical note (in italics)}

The staff surgeon asks for a "wishbone". The one available is not the one he wants. The scrub nurse explains the difficulty of changing the standing equipment order, referencing previous conversations they have had. The staff surgeon exclaims: "Well this is stupid, we're ordering new stuff and getting old stuff." The scrub nurse asks: "Anyone want to call CPD (the central processing department) AGAIN?" This particular equipment problem is not new to the team and yet it is not predicted prior to the case; rather, the communication arises at the moment of need, creating inefficiency of discourse and actions.

Tension:

Emotional responses to a communication failure; may ripple to other members/environments

In the instance regarding the wishbone (above), the circulating nurse, who is new to the division, responds that she will call CPD. The scrub nurse coaches her on what to say while the surgeon adds pointed suggestions. The circulating nurse is visibly anxious when she makes the call. When she hangs up the surgeon says "Well??"

The surgeon is irritated in response to a recurring resource problem that has not been addressed proactively. The frustration spreads to nursing and CPD.

Delay:

Communication failure results in a delay in the surgical procedure

In instances in which the surgical staff or resident has not been present for discussions of positioning or draping, these activities occasionally need to be redone to accommodate the particular needs of the surgical team.

Such rework efforts delay the commencement of a procedure, in addition to creating the effect of inefficiency in work practices.

Workaround:

Communication failure provokes a culturally accepted violation of an institutional regulation in order to maintain efficient workflow

After the patient has been anesthetized, the nurse tells the surgeon that the consent form used an abbreviation instead of the full procedure name, and adds that this is against regulations. The surgeon responds: "The key is, do you think he knew what he was coming for this morning?" The nurse assures: "Well, we didn't delay the case because of it...".

Members make a tacit agreement to work around the hospital regulation by assuming informed consent to ensure the $O R$ stays on schedule.

Resource waste:

Communication failure results in the use of equipment or personnel that is not required

Patient inconvenience:

Communication failure creates undue strain or imposition on patient

A cell saver, a critical and limited equipment resource, was ordered and set up. When the circulating nurse asked the surgical team when they would be using this equipment, the surgical fellow responded that they wouldn't be using it at all. Later the perfusionist enters and asks: "You don't need this cell saver?" to which the staff surgeon responds apologetically, "No, it's a cancer case. I should've told them that."

Had this information been transferred earlier, the equipment could have been dismantled and available if needed for another operating room theatre.

A patient has arrived to the operating room and is having IV lines inserted when the anesthesiologist communicates to the nurse that the patient's blood type information is "missing". The case preparation must be halted while the patient waits on the operating table for blood to be taken.

While "delay" is also a relevant effect, "patient inconvenience" acknowledges the added discomfort to the patient of delay in the OR environment rather than the holding area.

Procedural error:

Communication failure contributes to mistakes in

The anesthesia fellow inserts a triple lumen in the patient. The staff surgeon arrives and says: "I want a [Swan-Ganz line]." Pointing, he says: "That IV is not appropriate for a transplant." The anesthesia fellow, joined by the staff anesthesiologist, removes the triple lumen and replaces it with a Swan-Ganz line, a process that takes over 30 minutes.

This example illustrates the procedural error of the insertion of an inappropriate line necessitating removal and reinsertion, each step of which raises the risk to the patient. This error may be influenced by variables other than information transfer, such as the knowledge and supervision of the anesthesia fellow; however, the failure of the team to communicate about key procedural steps such as major lines allows other system weaknesses to perpetuate until an obvious threat to safety arises.

communication failures suggests critical aspects of team discourse that could be targeted for training initiatives to improve the communicative competence of the team. Each aspect is readily definable and easy to explain and demonstrate to team members. Furthermore, this classification is rooted in a theoretical framework that allows analysis of multiple dimensions of communication and how they interact to promote or undermine transfer of information and negotiation of procedural decisions in the operating room.

Although this study focuses on communication failures, our intention is not to suggest that they are distinct from the general organisation of the team's work context. On the contrary, communication failures are important in part because they can act as a signal of a problem originating elsewhere, in attitudinal or system processes. In the procedural error described in table 4, the staff surgeon questions the anaesthesia fellow too late (an occasion failure) and in the absence of the staff anaesthesiologist who had made the decision to insert a triple lumen line (an audience failure). These communication failures are not only contributing causes of the procedural error but also signals of other system issues such as trainee supervision.

As observational methods become more common in patient safety research, ethical considerations arise. In this study observers were ethically bound to intervene should they witness an immediate threat to patient safety. While no event requiring intervention arose, observers had to make judgments about what posed an "immediate threat". Such judgments were part of daily post-observation debriefing sessions but only surfaced for the one observer with extensive clinical expertise in the OR. In a few instances this observer intervened in lesser ways, such as when she was called upon to contact the clinical processing department for equipment urgently needed. While non-participant observation was the methodological framework for this research, the particular ethical considerations of patient safety research required a balancing of methodological and ethical goals.

In our findings a relatively small proportion (36.4\%) of communication failures resulted in immediate effects visible to the observers. This is probably a conservative depiction, reflecting the study design in which effects beyond the 
observation site-such as postoperative infections or equipment shortages in other OR theatres-were not available to the observer. However, the paucity of immediate effects is relevant to our understanding of communication practices in the operative setting, as it may encourage what Amalberti ${ }^{22}$ has conceptualized as the "migration" of practice from a regulated "safety zone" into an unregulated yet implicitly tolerated "zone of potential danger". A false sense of safety is produced when communication failure yields no immediate visible effect, which encourages further migration of communication practices until an event occurs that reveals the proximity of the team to the danger zone.

The invisibility of the effects of communication failure as well as the phenomenon of migration probably explain how the operating team has come to the status quo in which it is highly irregular for a surgeon, an anesthesiologist, and a nurse to meet and discuss a procedure before it commences. Compared with the expectations of other high risk organizations around team communication, this status quo is alarming.

In order to shift the status quo, research needs to render visible the effects of communication failure on both system processes and more distal health outcomes. Effects at both levels can then be used to construct a feedback loop ${ }^{23}$ for team communication practices. Clearly, communication failures are part of the wider system of processes and relations that produce the kinds of errors traditionally headlined in the safety literature, such as medication error or wrong sided surgery. But such sentinel events are often related to less visible communication failures that occur upstream in the system; in these instances the causal relationship between the communication failure and error may be remote, occluded by time and intervening events. When the relationship is a remote one, the process between communication failure and error may be populated with intermediate effects suggestive of higher environmental risk to safety. According to systems and error theory, increases in cognitive load, changes in routine, and emotional tension can ripen conditions for error to occur. ${ }^{23}$ In light of this, the inefficiency, delay, and team tension we observed may reflect a decreased resilience in the ability of the OR team to practice safely and prevent clinical errors.

Understanding the associative path between team communication practices, system processes, and health outcomes

\section{Key messages}

- Ineffective team communication is frequently at the root of medical error, but little is known about the specific ways in which communication fails.

- This observational study classified four types of communication failure: occasion (suboptimal timing), content (insufficiencies or inaccuracies), purpose (lack of resolution), and audience (gaps in group composition).

- Communication failures occurred in approximately $30 \%$ of team exchanges.

- The most frequently observed communication failures were exchanges that happened too late to be maximally useful and exchanges that were incomplete because relevant information was missing.

- One third of communication failures had immediate effects such as inefficiency and team tension. is a next step for work in this domain. Recent work has demonstrated a relationship between a model of surgical behavioral markers (including three generic communication items) and clinical outcomes. ${ }^{8}$ Our future research will continue this exploration of how teamwork impacts on outcomes by identifying "communication sensitive" outcomes and exploring their response to a team checklist intervention designed to reduce communication failure. The accrual of evidence regarding the precise relationship between team communication and health outcomes is a critical goal, for, although the status quo may be alarming, mandated change in communication routine is indefensible unless safety scientists can clearly demonstrate what difference such change will make.

\section{Authors' affiliations}

L Lingard, S Espin, S Whyte, G Regehr, G R Baker, R Reznick, J Bohnen, B Orser, D Doran, E Grober, University of Toronto, Toronto, Canada

This research was funded by the Canadian Institutes of Health Research (CIHR) and the Physicians of Ontario through the PSI Foundation. L Lingard is supported by a CIHR New Investigator Award; G Regehr is supported as the Richard and Elizabeth Currie Chair in Health Professions Education; B Orser is supported by an Ontario Ministry of Health Career Award.

\section{REFERENCES}

1 Kohn LT, Corrigan JM, Donaldson MS, eds. To err is human: building a safer health system. Washington, DC: National Academy Press, 2000.

2 Helmreich RL. On error management: lessons from aviation. BMJ 2000;320:781-5.

3 Helmreich RL, Davies JM. Team performance in the operating room. In Bogner MS, eds. Human error in medicine. Hillside, NJ: Erlbaum, 1994:225-53.

4 Joint Commission on Accreditation of Healthcare Organizations. Sentinel event statistics: 24 June 2003. Available online from, http://www.jcaho.org/ accredited+organizations/office+based+surgery/sentinel+events/ sentinel+event+statistics.htm (accessed 25 August 2003).

5 Chief Coroner, Province of Ontario. Inquest touching the death of Sanchia Bulgin: jury verdict and recommendations. July, 2001

6 Firth-Cozens J, Mowbray D. Leadership and the quality of care. Qual Health Care 2001; 10(Suppl II):ii3-7.

7 Firth-Cozens J. Multidisciplinary teamwork: the good, bad, and everything in between. Qual Health Care 2001;10:65-6.

8 Carthey J, de Leval M, Wright DJ, et al. Behavioral markers of surgical excellence. Saf Sci 2003;41:409-25.

9 Risser DT, Simon R, Rice MM, et al. A structured teamwork system to reduce clinical errors. In Spath PL, eds. Error reduction in healthcare. Chicago IL: AHA Press, 2000

10 Risser DT, Rice MM, et al. The potential for improved teamwork to reduce medical errors in the emergency department. Ann Emerg Med 1999:34:373-84.

11 Edmondson A, Bohmer R, Pisano GP. Learning new technical and interpersonal routines in operating room teams: the case of minimally invasive cardiac surgery. In: Neale MA, Mannix EA, Griffith TL, eds. Research on managing groups and teams:technology. Volume 3. Stamford, CT: JAI Press, 2000:29-51.

12 Lingard L, Reznick R, Espin S, et al. Team communications in the operating room: talk patterns, sites of tension, and implications for novices. Acad Med 2002;77:37-42.

13 Lingard L, Reznick R, DeVito I, et al. Forming professional identities on the healthcare team: discursive constructions of the 'other' in the operating room. Med Educ 2002;36:728-34.

14 Dwyer K. Surgery-related claims and the systems involved. Forum $2001 ; 21: 1-4$

15 Stout RJ, Salas E, Fowlkes JE. Enhancing teamwork in complex environments through team training. Group Dyn 1997;1:169-82.

16 US Food and Drug Administration. Anesthesia apparatus checkout recommendations, Available at: http://www.fda.gov/cdrh/humfac/ anesckot.pdf (accessed 13 January 2004).

17 Hammersly M, Atkinson P. Ethnography: principles in practice, 2nd ed. London: Routledge, 1995.

18 Creswell J. Qualitative inquiry and research design: choosing among five traditions. Thousand Oaks, CA: Sage Publications, 1998.

19 Lingard L, Haber RJ. Teaching and learning communication in medicine: a rhetorical approach. Acad Med 1999:74:507-10.

20 Burke K. A rhetoric of motives. Berkley: University of California Press, 1969.

21 ASHP. Therapeutic guidelines on antimicrobial prophylaxis in surgery. Am J Health Syst Pharm 1999;56:1839-88.

22 Amalberti R. The paradoxes of almost totally safe transportation systems. Saf Sci 2001;37:109-26.

23 Reason JT. Human error. New York: Cambridge University Press, 1990. 